\title{
Prototyping an Autonomous Face Controlled System using Raspberry-Pi on Wheelchairs
}

\author{
Shwetha H R, Nikhitha V Melige, Shabarish Reddy B S, Sujeeth U S, Sanjay D Gowda
}

\begin{abstract}
Quadriplegics are people who cannot use their extremities. Existing aiding devices are costly, complex, not user friendly and they are guardian dependent for movement. In this paper, a Raspberry Pi enabled wheelchair movement control by head movement detection is presented. A head movement detection technique based on Open - Computer Vision image processing is used which detects the user's facial movement in real time using the camera. Using this, the wheelchair motors are controlled and are driven in the indicated direction. Also, numerous sensors such as temperature sensor, pulse rate sensor, accelerometer sensor and fire sensor are added to monitor patient health, safety and well-being.
\end{abstract}

Keywords: Arduino Mega, Facial Landmark Technique, HOG, Open - CV, Raspberry Pi.

\section{INTRODUCTION}

Quadriplegic is a type of paralysis that affects all four limbs. Quadriplegia appears as a consequence of accident or age. Patients with such severe disabilities are unable to perform their daily actions, such as going to the bathroom, eating and moving around in space. Quadriplegic patients can retain certain level of freedom with the aid of medical devices that are designed. Raspberry Pi is an embedded system, it is low cost single-board computer system that can be used to reduce complexity of systems in real time applications. Previous studies and research have resulted in development of navigation and control system for an electrically powered wheelchair. The navigation and control was efficient, cost-effective, comfortable and easy to build with the components which were readily available [1]. The vision based control allows people using it to adapt and then command entire system at different levels of abstractions. Cluster is made up of distributed algorithms i.e. the performance monitor and network automation code that can

Manuscript received on October 24, 2021.

Revised Manuscript received on November 03, 2021.

Manuscript published on December 30, 2021.

* Correspondence Author

Shwetha H R, Assistant Professor, Department of Electronics and Communication Engineering, J. N. N. College of Engineering, Shivamogga (Karnataka), India.

Nikhitha V Melige, Department of Electronics and Communication Engineering, JNN College of Engineering, Shivamogga (Karnataka), India.

Shabarish Reddy B S, Department of Electronics and Communication Engineering, J. N. N. College of Engineering, Shivamogga (Karnataka), India.

Sujeeth U S, M.Tech, Department of VLSI Design, Vellore Institute of Technology, Chennai (Tamil Nadu), India.

Sanjay D Gowda, M.Tech, Department of VLSI Design, Vellore Institute of Technology, Chennai (Tamil Nadu), India.

(C) The Authors. Published by Blue Eyes Intelligence Engineering and Sciences Publication (BEIESP). This is an open access article under the CC BY-NC-ND license (http://creativecommons.org/licenses/by-nc-nd/4.0/) be executed from a single computer [2]. Usage of head movement analysis for the control of wheelchair is a plus point in case if the patient is paralyzed [3]. The captured head movement gestures can be used to generate control commands and hence making it possible to control wheelchair motors according to the user's head movement[5]. Open-CV algorithm is used to analyze the captured head movement and through this data, motors are controlled, and wheelchair is moved [6]. Open-CV (Open-Source Computer Vision Library) is an open-source computer vision and machine learning software library. It is a cross-platform library that can be used to develop real-time computer vision applications. The library consists of programming functions mainly aimed at real time computer vision. The main focus is on real-time image processing. Computer vision is the construction of explicit, meaningful descriptions of physical objects from an image, the output of computer vision is a description or an interpretation of structure in 3D scene. Facial landmark detection technique is used to detect key landmarks on the face and track them. Facial landmark detector implemented inside d-library produces $68(\mathrm{x}, \mathrm{y})$-coordinates that map to specific facial structures. These mappings are obtained by training shape predictor on the labeled iBUG 300-W dataset. HOG (Histogram of Oriented Gradients) is a feature descriptor that allows to extract features from image data. Main focus of HOG descriptor is on the structure or the shape of an object.

In this proposed work, a microprocessor, Raspberry $\mathrm{Pi}$ is used to enable a standard electric wheelchair control by facial movement detection. This idea utilizes a camera attached to the raspberry pi to capture facial movement. Flex sensor is used to activate camera, which captures the facial movement at the rate of 30 frames per second. Open - CV algorithm is used along with the facial landmark technique to compute the facial movement. Based on the facial movement, Raspberry $\mathrm{Pi}$ controls the electric motors of the wheelchair. If the movement is right then the chair is moved right and so if the if movement is left the chair is moved left, similarly for up and down it is front and back. In addition to this numerous sensor are added to monitor patient's health and well-being. Pulse sensor and temperature sensor are used to monitor patient health continuously, and an alert e-mail is sent accordingly in case of variation in patient's health to guardian. Fall detection is observed using accelerometer sensor and surrounding is alerted if in case the wheelchair tumbles. Fire alert is also added to the wheelchair. Home automation is done using Bluetooth, thus providing control to home appliances form the seat.

\section{Published By:}

Blue Eyes Intelligence Engineering and Sciences Publication (BEIESP)

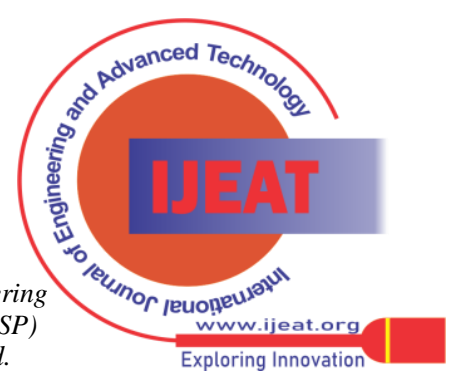




\section{Prototyping an Autonomous Face Controlled System using Raspberry-Pi on Wheelchairs}

\section{METHODOLOGY}

Raspberry Pi and Arduino are the central control system of the proposed work. Entire control and coordination are done using them. All the other sensors are placed around them. Aim of the proposed work is to "Provide an automated wheelchair control using head movement detection to assist people with disabilities. Also monitor their health using pulse sensor and temperature sensor. To check if the wheelchair tumbles using 3-axis accelerometer sensor and provide control to electric appliances in the house through their seat."

As shown in the figure 1, a Raspberry Pi system is used which enables wheelchair control by head motion. A Flex sensor is used to activate Camera. It is attached to the Raspberry $\mathrm{Pi}$ to capture head movement. The camera captures the head movement and data will be sent to the Raspberry Pi to analyze the frame. The Open-CV algorithm is used to compute the head movement. Based on the movement the Raspberry Pi controls the Electric motors of the Wheelchair. Moving face left or right will make the wheelchair rotate left or right, similarly up and down will move the wheelchair forward or backward respectively.

In addition to this, numerous sensors are added. Fall detection is analyzed using 3-axis accelerometer sensor, if the chair tumbles an alarm gets triggered and an email will be sent to caretaker stating that the person has fallen. Patient's health is monitored using pulse sensor and temperature sensor, an alert email will be sent to the guardian if the person has a high pulse rate or high temperature. A fire detector is kept in the wheelchair to monitor fire accidents. Ultrasonic sensor is kept in the front side of wheelchair to check if there are any obstacles present on the way and then stop the wheelchair movement if it is present.

Home automation is added in order to control the home appliances wireless through Bluetooth module. Control for few electronic components such as light and fan is enabled, it can be accessed using switches provided on wheelchair.

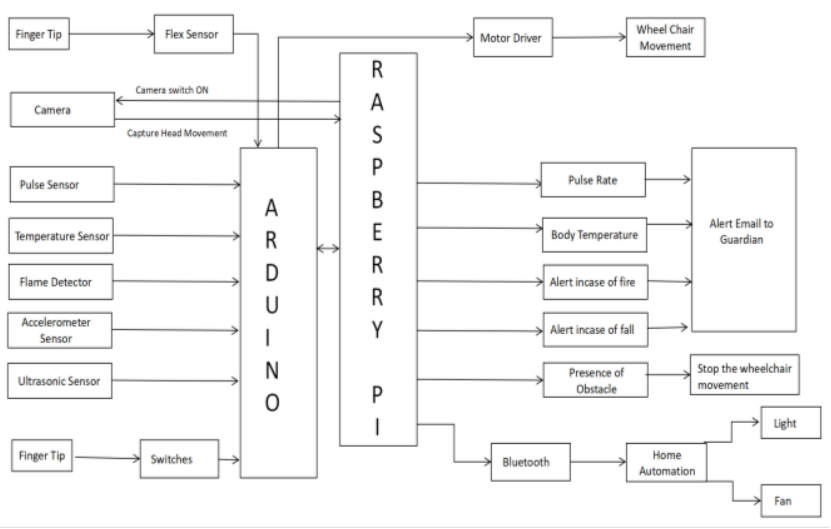

Figure 1: Block diagram of the proposed work

The below figure 2 shows the entire flow of the proposed work. Once the system gets started, flex sensor must be activated which is placed at the tip of the index finger. On activation camera starts working and detects the face and a frame is drawn around it for further movement analysis. The system checks if there are any obstacles present on the way using ultrasonic sensor and then the wheelchair starts moving in the indicated direction, i.e through the command given through face movement. If there are any obstacles present on the way, then the wheelchair doesn't move. Pulse sensor, temperature sensor, flame sensor and accelerometer sensor are activated once when the system is switched on. These sensors continuously check for the output and in case if the output from these sensor is high then an alert email is sent to the corresponding guardian. If the sensors output is low then there would be no changes and the loop continues. Inputs are drawn from the switches which are placed on the arm rest of wheelchair and from it home automation is enabled Command is sent to the controller and then from bluetooth module light and fan can be switched on.

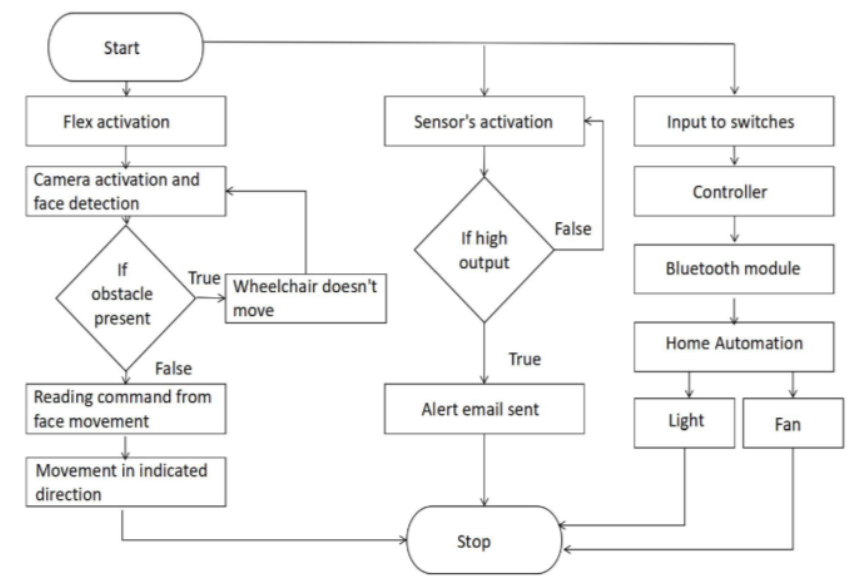

Figure 2: Flow diagram of the proposed work

Following is the algorithm for this proposed work,

\section{A. Face Movement Analysis}

1. Start the process.

2. Activation of flex.

3. Camera Activation and face detection using Open CV algorithm.

4. Movement analysis in each frame.

5. Check if obstacle is present ahead.

6. If yes stop the wheelchair.

7. If no direct the motor to move accordingly.

8. Hence wheelchair movement in indicated direction.

\section{B. Health Monitoring}

1. Start the process.

2. Activating the sensors.

3. Reading input from the patient at regular intervals.

4. If senors output is high alert email sent to recipient

5. If sensors output is low process repeats.

\section{Home Automation}

1. Start the process.

2. Reading input from switches.

3. Sending them to Bluetooth module.

4. Switching the light and fan on accordingly.

\section{RESULT AND DISCUSSION}

After execution of the Open-CV algorithm, face was detected and the movement was analyzed. The movement of eyes, nose and mouth, face as a whole decides the wheelchair movement. A green color box is drawn around the nose and movement of the face with respect to nose is read and the output is displayed, it is as shown figure 3.

Published By:

Blue Eyes Intelligence Engineering and Sciences Publication (BEIESP)

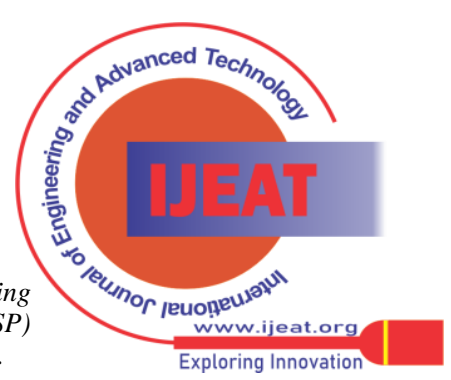




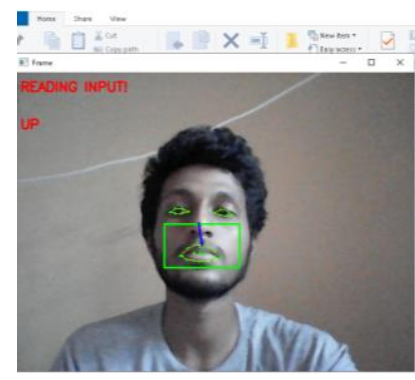

(a)

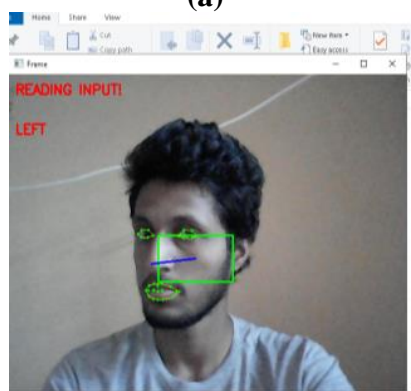

(c)

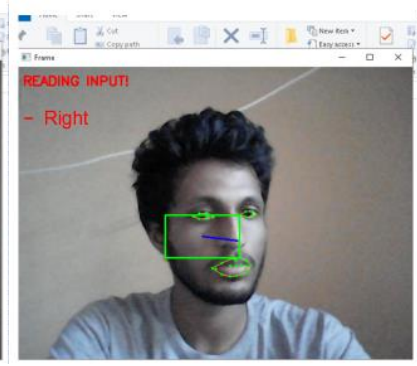

(b)

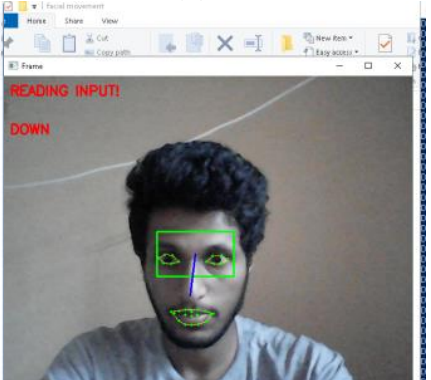

(d)
Figure 3: Simulated Results: (a) Upward Movement (b) Right Movement (c) Left Movement (d) Downward Movement

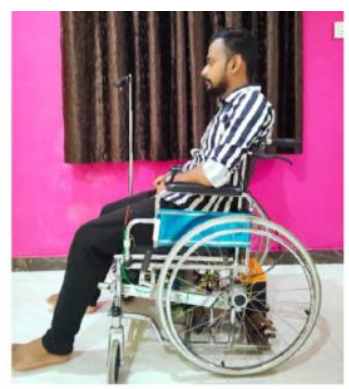

Figure 4: Wheelchair setup

Wheelchair set up for the quadriplegics is as shown in figure 4. It consists of various components arranged accordingly. Figure 5 shows the pictures of sensors placed on the wheelchair. Pulse sensor reads the pulse rate of the patient continuously. Temperature sensor reads the body temperature of the patient. Both of these sensors are placed on the arm rest of the wheelchair. The accelerometer sensor monitors steadiness of the wheelchair. All the sensors will read the input once the system gets started and if the output of these sensors are high then, an alert email is sent to the respective recipient. The values are read on regular intervals and hence enabling continuous monitoring of health. Fire detector is placed under the wheelchair seat, it monitors fire accidents and ensures safety. If in case fire is detected an alert email is sent. The Ultrasonic sensor is kept in front of the wheelchair to check if the path ahead is free from obstacles or not, if any obstacle is present then the wheelchair doesn't move. It will remain still.

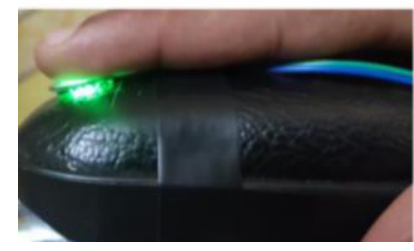

(a)

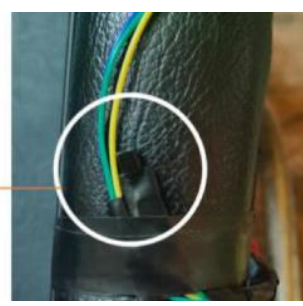

(b)

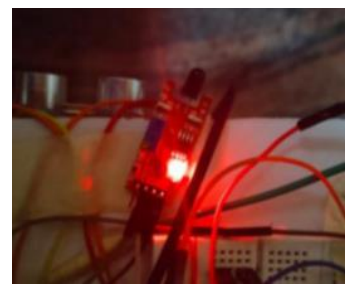

(d)

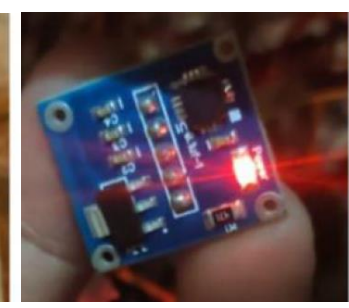

(c)

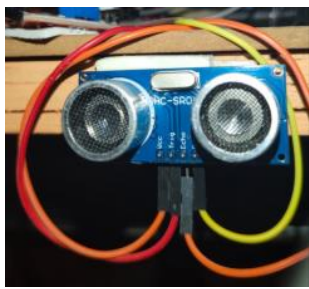

(e)
Figure 5: Sensors placed in the wheelchair: (a) Pulse Sensor (b) Temperature Sensor (c) Accelerometer Sensor (d) Fire Sensor (e) Ultrasonic Sensor

'Email alert' is enabled to alert the guardian when there is health variation. If the read value crosses the threshold value or the pre-fixed value then, an email according to the drafted format will be sent. Figure 6a and 6b shows the alert email snapshot sent high body temperature and for high pulse detected. Figure 6c and 6d shows alert email snapshot sent when flame and fall is detected.

Figure 7 shows the home automation setup. Through the switches fan and light at a distance could be turned on or off. Input read from the switches is given to the controller and this data is sent to the Bluetooth module at a distance and from there the fans and light which are plugged in are switched on. Hence home automation is enabled.

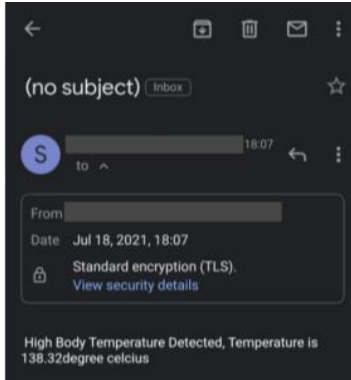

(a)

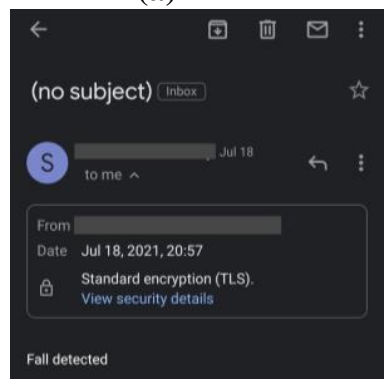

(c)

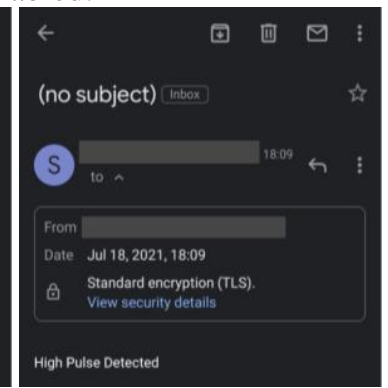

(b)

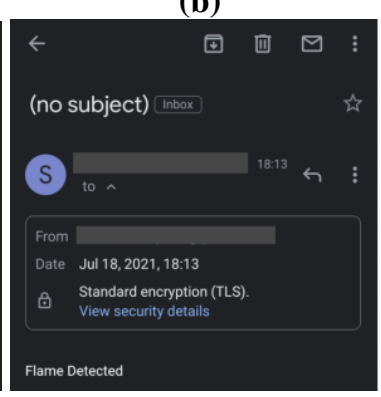

(d)
Figure 6: Alert Message to Guardian through Email: (a) High Temperature (b) High Pulse (c) Fall Detection (d) Flame Detection

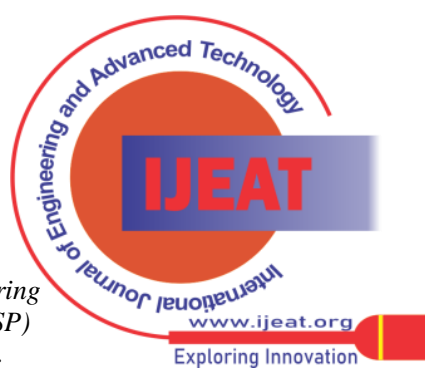




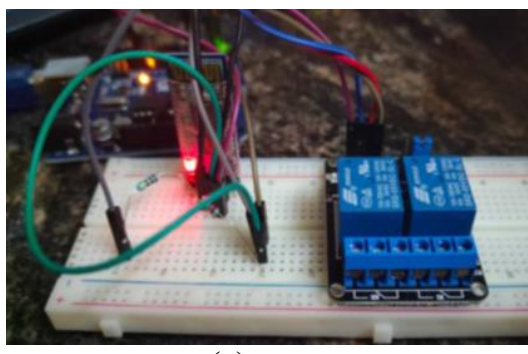

(a)

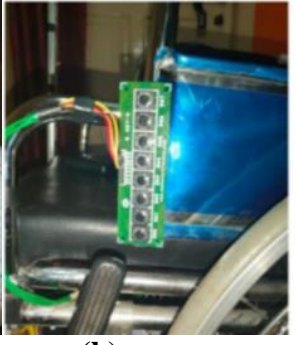

(b)
Figure 7: Home automation Setup: (a) Bluetooth Setup (b) Switches

\section{CONCLUSION AND FUTURE SCOPE}

The design and implementation of a wheelchair design for quadriplegic patients. This developed system provides mobility for disabled people who have restricted movements. An Open-CV algorithm is used to detect and analyze the movement. Wheelchair motors are controlled using the head movement and are moved according to the indicated direction. Health monitoring of the patient can be achieved with the temperature and pulse sensor. Well-being of the system can be monitored using the accelerometer sensor and fire sensor. Home automation, fan and TV control is achieved using the bluetooth setup. This design is easy to use, low-priced and user-friendly device which serves multiple purposes. It is also cost effective, comfortable and easy to build with the components which are readily available. Future research will be focused on SMS being sent instead of e-mail alert. An alarm sound on fire to alert the surrounding. Ultrasonic sensor can be placed on all the four sides to regulate the movement in all the directions. Speed control gears can be deployed for smooth movement of the chair.

\section{ACKNOWLEDGMENT}

We are thankful to 'Karnataka State Council for Science and Technology, 44th series Student Project Program (SPP): 2020-21' for granting us fund and supporting in making the model of this proposed idea. We acknowledge 'Financial Assistance for Innovative Projects for Final Year undergraduate students for the year 2020-2021', for providing us fund and in helping us complete the model.

\section{REFERENCES}

1. "Smart Navigation and Control System for Electric Wheelchair" - Bader Dakhilallah Samran Alrashdi, K. Prahlad Rao1 and Naif D. Alotaibi in American Journal of Engineering Research (AJER), Volume-8, Issue-4, 2019

2. "Human-Machine Interface for a Smart Wheelchair" - Amiel Hartman and Vidya K. Nandikolla in Hindawi , Journal of Robotics, Volume 2019, Article ID 4837058, 2018

3. "A Wheelchair Control System Using Human-Machine Interaction: Single Modal and Multi Modal Approaches" - Mohamed Shahin, Alaa Tharwat, Tarek Gaber* and Aboul Ella Hassanien in De Gruyter Journal of Intelligent Systems, Volume 28: Issue 1, 2017

4. "Development of Smart Wheelchair System for a User with Severe Motor Impairment" - Mohd Razali Md Tomaria, Yoshinori Kobayashia, Yoshinori Kuno in Elsevier Ltd International Symposium on Robotics and Intelligent Sensors, 2012

5. "Head gesture recognition for hands-free control of an intelligent wheelchair" - Pei Jia , Huosheng H. Hu, Tao Lu and Kui Yuan in Industrial Robot: An International Journal, Volume 34, Number 1, 2007

6. "Real Time Face Detection and Tracking Using Open-CV" - Prof. P Y Kumbhar, Mohammad Attaullah, Shubham Dhere, Shivkumar Hipparagi in International Journal for Research in emerging science and technology, volume-4, issue-4, 201, 2017

\section{AUTHORS PROFILE}

Shwetha $\mathbf{H} \mathbf{R}$ received B.E. degree in electronics and communication from Visvesvaraya Technological University, Karnataka, India, in 2005 and M.Tech. degree in VLSI design and embedded system from (SJCE) VTU,

Karnataka, and now pursuing Ph.D. in microelectronics and nanotechnology from Visvesvaraya Technological University, Karnataka, India. She is currently Assistant Professor in Department of Electronics and Communication Engineering, J N N College of Engineering, Shivamogga.
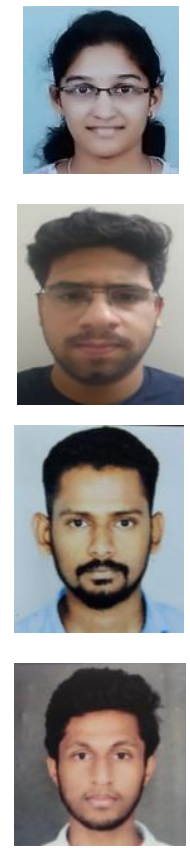

Nikhitha V Melige, has completed her under graduation in Electronics and Communication from J N N College of Engineering under VTU, Shimoga in the year 2021. She is now currently working in TCS as Assistant System Engineer- Trainee.

Shabarish Reddy B S has completed his engineering from J N N College of Engineering under VTU, Shimoga in the filed of Electronics and Communication in the year 2021.

Sujeeth U S has completed his engineering in the filed of Electronics and Communication from J N N College of Engineering under VTU in 2021. And is currently pursuing M.Tech in the filed of VLSI Design in Vellore Institute of Technology, Chennai.

Sanjay D Gowda has completed his engineering in the filed of Electronics and Communication from $\mathrm{J} \mathrm{N} \mathrm{N}$ College of Engineering under VTU in 2021. And is currently pursuing M.Tech in the filed of VLSI Design in Vellore Institute of Technology, Chennai.

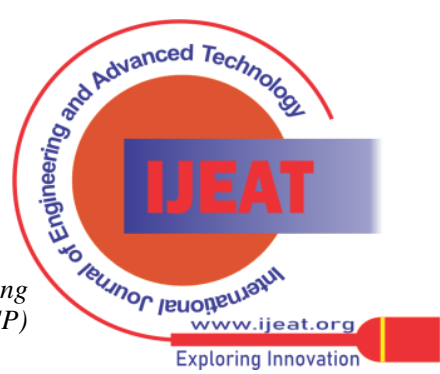

